\title{
Neurology for the twenty-first century
}

\author{
Khalid Hamandi
}

Khalid Hamandi

MB BS BSC MRCP, Specialist Registrar in Neurology, Gloucester Royal Hospital

This conference was held at the Royal College of Physicians on 4 July 2002

Clin Med JRCPL 2002;2:578-80

\section{Introduction}

This was the first joint meeting organised by the Royal College of Physicians and the Association of British Neurologists. Public expectation, clinical governance, and continued advances in disease therapy should drive much needed expansion in British neurology. In the last decade of the twentieth century, major advances in molecular science and imaging techniques took place, leading to greater understanding of neurological disease. These advances go hand in hand with traditional clinical methods.

\section{Acute paralysis}

The diagnosis of an acute symmetrical weakness is one that relies heavily on the history and examination findings. The site of the lesion is deduced by the presence of upper or lower motor neurone signs, cranial nerve involvement, sensory disturbance and sphincter dysfunction.

Guillain Barre Syndrome (GBS) presents with flaccid weakness, areflexia and sensory symptoms with less demonstrable sensory loss. Respiratory and bulbar weakness can lead to ventilatory failure and aspiration pneumonia. Spinal cord disease can have a similar pattern in the acute setting with flaccid limb weakness and areflexia during the spinal shock stage, before development of hypertonia, hyperreflexia and extensor plantars. An ascending clinical pattern in myelitis can cause confusion with GBS. Early bladder involvement and a sensory level should alert the physician to spinal cord involvement.

Vasculitic neuropathy rarely presents with a symmetrical tetra- or paraparesis. Difference in density in motor or sensory deficit in different peripheral nerve territories is an important clue. Other features include the presence of systemic symptoms, rash, abnormal urinalysis, raised inflammatory indices and positive anti-neutrophil cytoplasmic antibody (ANCA). An affected sural nerve, demonstrated neurophysiologically, is most likely to reveal histological abnormalities on nerve biopsy. Early diagnosis and treatment with high dose steroids and cyclophosphamide is important to prevent further, permanent deficits. Rarer causes of acute neuropathy include Lyme disease and porphyria.
Neuromuscular junction disorders and primary muscle disease should not be forgotten in the patient with acute paralysis, particularly in the absence of sensory abnormalities. Features suggesting myasthenia gravis include ptosis, weakness of neck muscles and early respiratory weakness. Botulism causes autonomic disturbance, pupil abnormalities and a descending weakness. Muscle pain and a markedly raised creatine kinase point toward inflammatory muscle disease.

Supportive care and early involvement of high dependency or intensive care units are essential in the treatment of acute neuromuscular weakness. Measures include prophylaxis against venous thrombosis, monitoring and ventilatory support, maintenance of adequate nutrition, and monitoring and treatment of cardiac and autonomic disturbance. In the treatment of GBS, trials show benefit with plasma exchange or intravenous immunogobulin (IVIG) against placebo but no difference between them. For practical reasons IVIG is now standard treatment. Multidisciplinary care, physiotherapy and rehabilitation remain important.

\section{Prion disease: mechanisms and prospects for treatment}

Sporadic, inherited, and transmissible forms of prion disease exist - Creutzfelt Jacob disease (CJD), Gerstmann-Sträussler Sheinker (GSS), familial fatal insomnia (FFI) and Kuru in man, scrapie in sheep, and bovine spongiform encephalopathy (BSE) in cattle. Prion disease is unique in that the transmissible agent, prion protein $(\operatorname{PrP})$ contains no nucleic acid. It is a disease of protein folding or processing, the study of which may provide a greater understanding of other neurodegenerative diseases, for example Alzheimer's and Parkinson's disease.

$\mathrm{PrP}$ is expressed in all mammalian species. Contact of the host's encoded protein $\mathrm{PrP}^{\mathrm{c}}$ with an abnormal infecting isoform ( $\mathrm{PrP}^{\text {sc (scrapie) }}$ ) leads to an alteration in its structure to that of the abnormal isoform with accumulation in brain and other tissue, thus explaining infectivity and propagation without nucleic acid. CJD is rare (one per million). Most cases are sporadic. It can be inherited as autosomal dominant or acquired. Identified modes of transmission include iatrogenic, following treatment with pituitary-derived growth hormone, corneal and 
dural grafts, cannabalistic rituals in Papua New Guinea leading to Kuru, and most recently, variant (v)CJD from BSE-infected cattle. The prion strain in $\mathrm{VCJD}$ is indistinguishable from that causing BSE, providing compelling evidence that direct transmission has occurred. To date (July 2002), there have been 124 cases of vCJD in the UK. The lymphoreticular system is heavily involved, and tonsil biopsy can be used to make the diagnosis in life.

Around 30 genetic mutations have been identified in inherited CJD. In addition there exist 'innocent' polymorphisms. That at codon 129 affects disease susceptibility, encoding either methionine or valine. Fifty per cent of the population are heterozygous at this locus which is protective against iatrogenic and familial CJD, and Kuru, with later onset disease. All vCJD cases to date are homozygous for methionine at this position.

Evidence from molecular science supports current theories in disease process. PrP can refold to different isoforms. Under normal circumstances the majority exists in $\boldsymbol{\alpha}$ helical form $\mathrm{PrP}^{\mathrm{c}}$. A small amount of $\operatorname{PrP}^{\mathrm{c}}$ proceeds, via a reversible reaction involving a high degree of protein unfolding to $\boldsymbol{\beta}$ sheets. This is prone to fibrillar aggregation that is insoluble and protease resistant. Contact with infecting $\operatorname{PrP}^{\text {sc }}$ (or $\boldsymbol{\beta}$ - $P r P$ ) acts as a seed for formation of further $\boldsymbol{\beta}$ sheets and irreversible fibrillar formation, thus propagating the reaction further.

A number of strategies for treatment are being developed. Licensed drugs could be tested in animal models, to identify those that slow the disease process as more definitive treatments are being developed. For such treatment to be acceptable, early diagnosis prior to the onset of major disability would be needed. Other strategies include the development of antibodies or vaccines that block prion replication or novel drugs that bind to and stabilise $\mathrm{PrP}^{\mathrm{c}}$ preventing unfolding and $\boldsymbol{\beta}$-sheet formation. High throughput screening of known compounds is currently underway with collaboration between the MRC Prion Unit and GlaxoSmithKline.

Recent publicity arose after publication of experiments that show chlorpromazine and quinacrine inhibit $\mathrm{PrP}^{\mathrm{sc}}$ formation in vitro. Premature or 'unofficial' access to potential therapies could lead to inappropriate use or failure to recognise benefit. It is vital to assess new therapies objectively. The MRC Prion Unit aims to put in place the structure to facilitate research and diagnostics and to enable durable double blind clinical trials to take place when treatments become available.

\section{New anti-epileptic drugs}

Eight new anti-epileptic drugs (AEDs) have been licensed in the UK since the late 1980s, and others are under development. The goals of complete freedom from seizures and absence of drug side-effects remain elusive. All AEDs are effective in partial onset seizures, carbamazepine being the most accepted. Sodium valproate tends to be favoured in generalised seizures, although a number of AEDs are effective. Awareness of teratogenicity and hormonal effects of AEDs in women with epilepsy has increased; currently lamotrigine is favoured in this group.

New AEDs are initially licensed as add-on therapy to one of

\section{Conference programme}

\author{
- A critical assessment of NICE \\ Professor J Collier, St George's Hospital Medical School, \\ London
}

I Neurological intensive care

Dr R Howard, St Thomas' Hospital, London

\section{Acute paralysis}

Dr M Donaghy, University of Oxford
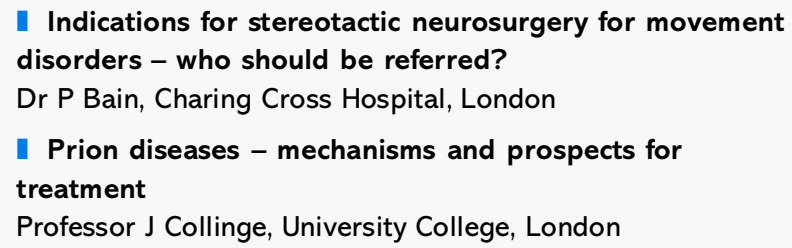

- Future neurological services

Professor C Warlow, President, Association of British Neurologists

- Prospects for treatment of acute stroke

Professor P Sandercock, Western General Hospital, Edinburgh

- Secondary prevention of stroke

Dr P Rothwell, Radcliffe Hospital, University of Oxford

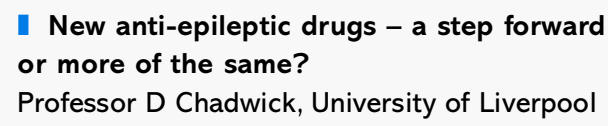

- Evidence-based treatment of multiple sclerosis

Professor D H Miller, Institute of Neurology, London

the standard AEDs. Their efficacy is measured as a reduction in seizure frequency. In general, the more potent drugs seem to have more adverse effects, as assessed by withdrawal rates. Drug combinations with different mechanisms of action may be beneficial. Comparisons between new and standard therapies in terms of efficacy, adverse events (dose related, idiosyncratic, chronic and teratogenic), ease of use, drug-drug interactions and cost, are important. Before launching new drugs, pharmaceutical industry studies (usually comparing drug with placebo) are aimed at licensing rather than clinical usefulness, and therefore longer-term, head-to-head trials are necessary. Information on psychosocial and health economic outcomes also needs to be collected. In an attempt to answer these questions a national prospective study, SANAD (Standard and New Anti-epileptic Drugs), funded by NHS R\&D, was set up. This compares sodium valproate and carbamazepine with new AEDs and is due to report in 2004. Post-marketing surveillance is essential to assess possible longer-term adverse effects of these drugs. Vigabatrin causes irreversible visual field constriction in $40 \%$ of patients, a chronic adverse event that was not recognised until some years after its release, and it no longer has a place in the treatment of adults.

The cost of new AEDs is not small. If they are to be used as a first line treatment, superior efficacy and tolerability, or equivalent efficacy and better tolerability, needs to be demonstrated. 
Concerns about chronic toxicity and safety for women during child-bearing years also need to be addressed.

\section{Evidence-based treatment in multiple sclerosis}

Treatment of multiple sclerosis (MS) with disease modifying therapy has been the subject of much controversy over recent years. MS is a chronic disease with a clinical duration over decades. The natural history of the disease with a relapsing remitting phase, usually followed by a secondary progressive phase, is well recognised. Primary progressive and a progressive relapsing form also exist. Pathological findings are of multifocal inflammatory demyelinating lesions in the central nervous system. In the progressive phase there is axon loss and neurodegeneration. This has implications for disease modifying therapy that only targets the inflammatory component.

Several trials in the past decade showed that treatment with interferon and glatiramer acetate (a synthetic peptide) reduces the number of relapses by one-third in relapsing remitting disease, over two to three years. There was less disease activity on magnetic resonance imaging (MRI), and fewer gadoliniumenhanced lesions (an objective marker of pathology representing areas of blood drain barrier breakdown, inflammation and demyelination) in the treated groups. Some evidence suggested less progression to disability on a 10-point rating scale (the Kurtzke expanded disability status scale).

There are still major unresolved issues in the clinical and cost effectiveness of interferon and glatiramer acetate. The duration of the trials was short relative to the disease. The natural history in any individual is unpredictable. There is a strong subjective element to relapses, albeit with some objective measures. Although early relapse frequency and severity is partially related to long-term disability, factors other than inflammation appear to affect outcome. Disability rating scales used in the trials have a low sensitivity and poor reproducibility and do not reflect all aspects of disease. Effects of treatment on inflammatory lesions seen on MRI are not accompanied by similar clinical efficacy. This is also the case in trials with immunosuppressive agents mitaxantrone and campath-1H (a lymphocyte depleting monoclonal antibody). Novel MRI techniques that include measurements of neurodegenaration and brain atrophy as well as inflammation are required.

After negotiation between the Department of Health, the National Institute for Clinical Excellence (NICE), pharmaceutical companies and MS support groups, a 'risk sharing scheme' was introduced in May 2002. Interferon and glatiramer acetate can be prescribed on the NHS by consultant neurologists, with close long-term monitoring of treatment effects.

Future modes of treatment are likely to involve immunosuppresive and immunomodulatory therapy, perhaps in combination. Natulizumab is a monoclonal antibody against a lymphocyte adhesion molecule $\boldsymbol{\alpha} 4 \boldsymbol{\beta} 1$ integrin. It prevents trafficking of lymphocytes across the blood brain barrier. Results of phase 2 studies are promising and phase 3 trials are underway. Strategies for neuroprotection, remylination and repair are being devel- oped.

\section{Conclusion}

The cost benefit of new treatments was a recurring theme at this and many other neurological conferences. Robust clinical trials are needed to address conflicts between the commercial interests of the pharmaceutical industry and scientific freedom. There is an opportunity to gain greater understanding of disease process through these therapeutic advances. 